\title{
Waves stranded at sea: bound states in the continuum in a strong coupling regime
}

\author{
Shaimaa I. Azzam and Alexander V. Kildishev \\ Purdue University, School of Electrical and Computer Engineering and Birck Nanotechnology Center, West Lafayette, Indiana, \\ United States
}

What, exactly, is a bound state in the continuum (BIC)? A BIC is an unconventional state that remains localized even upon its coexistence with the continuum of radiating modes. In theory, a BIC is characterized by the infinite radiative quality factor and zero linewidth. ${ }^{1}$ Amongst the various types of BICs, the so-called Friedrich-Wintgen BIC arises due to the destructive interference of two resonances coupled to the same radiation channel. Once the eigenvalue of one of the resonances becomes purely real, the state turns into a BIC. Generally, Friedrich-Wintgen BICs lie in the vicinity of the frequency crossings of the uncoupled resonances.

Recently, the exploration of BICs in the strong coupling regime has gained increasing attention to diverse systems such as coupled photonic-plasmonic devices, ${ }^{2}$ D materials, ${ }^{3}$ and low-contrast dielectric gratings. ${ }^{4}$ In some of these systems, strong coupling between resonances of different origin has been studied such as the interaction of plasmonic modes and the optical waveguide modes of a hybrid system ${ }^{2}$ or the coupling of excitons and polaritons in a photonic crystal coupled to $\mathrm{WSe}_{2}$ monolayers. ${ }^{3}$ Such heterogeneous systems offer great opportunities for realizing new hybrid quasiparticles with rich physics. The Friedrich-Wintgen BICs arising in the vicinity of the avoided crossing of the resonance lead to very high-quality resonances.

In this issue of Advanced Photonics, Bogdanov et al. ${ }^{5}$ reveal the formation of the Friedrich-Wintgen BICs in a tiny resonant system as small as a nanoparticle (see Figure). First, the authors explore quasi-BIC states that arise due to the strong coupling between two classical phenomena (Mie-like and Fabry-Perot-like modes) supported by such a resonator. They then demonstrate that surprisingly high quality factors and strong light localization can be supported by a subwavelength cylinder due to the excitation of the quasi-BIC state. They also analytically examine the curious, tight relationship between the existence of the quasi-BIC states and the peculiarities of the Fano asymmetry parameter in the strong coupling regime, showing that the Fano asymmetry parameter becomes singular at the quasi-BIC point. The team confirmed this unusual strong coupling experimentally. By measuring the scattering cross-section of a cylindrical resonator filled with water, the authors are able to capture the "avoided crossing" (a direct confirmation of the case of strong coupling ${ }^{6}$ ) as a function of the cylinder aspect ratio.

The innovative ideas proposed and experimentally demonstrated by Bogdanov et al. could serve as a new paradigm for rapidly projecting the nascent BIC methodology onto numerous optical phenomena and applications that rely on high field localization and high quality factors, which now can be achieved with a single-particle resonator at the subwavelength scale, something that was difficult to achieve previously. On-a-chip applications such as low-threshold lasing and high-harmonic generation are examples of the many possibilities that would make use of such an exciting ultracompact novel nanophotonic platform.

○ 2019 SPIE and CLP. [DOI: 10.1117/1.AP.1.1.010503]

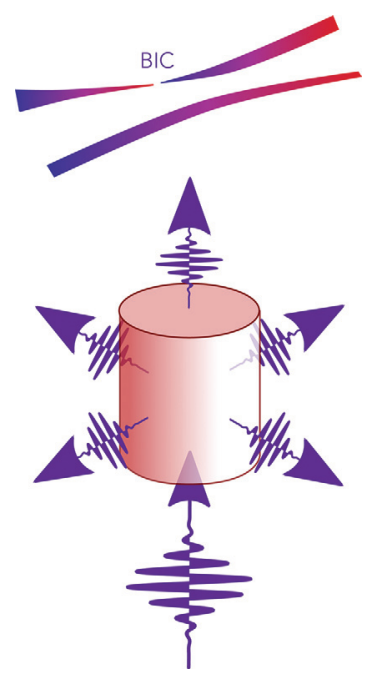

$\mathrm{BIC}$ in a nanoparticle in the strong coupling regime.

\section{References}

1. C. W. Hsu et al., "Bound states in the continuum," Nat. Rev. Mater. 1, 16048 (2016).

2. S. I. Azzam et al., "Formation of bound states in the continuum in hybrid plasmonic-photonic systems," Phys. Rev. Lett. 121(25), 253901 (2018).

3. K. L. Koshelev et al., "Strong coupling between excitons in transition metal dichalcogenides and optical bound states in the continuum," Phys. Rev. B 98(16), 161113 (2018).

4. E. N. Bulgakov and D. N. Maksimov, "Avoided crossings and bound states in the continuum in low-contrast dielectric gratings," Phys. Rev. A 98(5), 053840 (2018).

5. A. Bogdanov et al., "Bound states in the continuum and Fano resonances in the strong mode coupling regime," Adv. Photonics 1(1), 016001 (2019).

6. L. Novotny, "Strong coupling, energy splitting, and level crossings: a classical perspective," Am. J. Phys. 78, 1199-1202 (2010).

Shaimaa I. Azzam is working towards her $\mathrm{PhD}$ in electrical and computer engineering at Purdue University. She received her MS and BS degrees in electrical engineering in 2014 and 2011, respectively. Her research interests include metasurfaces, lasers and nanolasers, multiphysics time and frequency domain photonics simulations, passive and active plasmonics, and topological states of light.

Alexander V. Kildishev works in the field of the theory and numerical models for nanophotonics. He has had a number of breakthrough results on negative refractive index metamaterials, optical artificial magnetic structures, loss compensation in metamaterials, plasmonic nanolasers, and optical metasurfaces, as well as optical cloaks and hyperlenses. His current h-factors are WEB of Science-50, Google Scholar-60. He is a coinventor of 14 issued and pending patents (7 U.S. patents). 